\title{
Ecologia e variação espacial de Naupactus lar Germar (Coleoptera, Curculionidae, Entiminae) no Parque Nacional da Restinga de Jurubatiba, RJ
}

\author{
Cristina de Oliveira Araujo ${ }^{1,2}$, Vivian Flinte ${ }^{1,2}$, Margarete Valverde de Macêdo ${ }^{1}$ \& Ricardo Ferreira Monteiro ${ }^{1,2}$
}

\author{
${ }^{1}$ Laboratório de Ecologia de Insetos, Depto. de Ecologia, IB, UFRJ. Caixa Postal 68020, 21941-590 Rio de Janeiro-RJ, Brasil. \\ crisaraujo@biologia.ufrj.br; flinte@biologia.ufrj.br; mvmacedo@biologia.ufrj.br; monteiro@biologia.ufrj.br \\ ${ }^{2}$ Programa de Pós Graduação em Ecologia, UFPR.
}

\begin{abstract}
Ecology and spatial variation of Naupactus lar Germar (Coleoptera, Curculionidae, Entiminae) at the National Park of Restinga de Jurubatiba, State of Rio de Janeiro. The present work aimed to describe the pattern of spatial variation of $N$. lar at the National Park of Restinga de Jurubatiba and to study aspects of the species ecology and behavior. Bimonthly surveys were conducted on 120 Byrsonima sericea plants, one of its main hosts, from February 2003 to May 2004, in four different areas of the park. Four color morphs were found (yellow, pink, orange and white), but not all occurred together in the same area. Almost all adult males observed in field were yellow, except for one orange individual. Females, however, showed all cited color morphs. Tests in laboratory showed that individuals of different colors and/or from different sites mated normally among themselves, indicating that there is no mechanical or behavioral barrier preventing copulation among them. Adults of $N$. lar occurred throughout the year, showing an abundance peak between October and February, and lower numbers between April and August. Both percentage of plant occupation and density of individuals on plants followed the pattern of the populational fluctuation. In this way, it seems that $N$. lar presents one reproductive season per year, between October and February, period of rainy season and production of new leaves of the food plants.
\end{abstract}

KEYWORDS. Byrsonima sericea; color variation; life cycle; spatial distribution; populational fluctuation.

RESUMO. Ecologia e variação espacial de Naupactus lar Germar (Coleoptera, Curculionidae, Entiminae) no Parque Nacional da Restinga de Jurubatiba, RJ. O presente trabalho teve como objetivos descrever o padrão de variação espacial na coloração de $N$. lar no Parque Nacional da Restinga de Jurubatiba e estudar aspectos da sua ecologia e de seu comportamento. O trabalho foi realizado de fevereiro de 2003 a maio de 2004, em excursões bimestrais a quatro diferentes áreas do parque, vistoriando um total de 120 plantas de Byrsonima sericea, um de seus principais hospedeiros. Foram encontrados quatro tipos de coloração (amarelo, rosa, laranja e branco), sendo que nem todas as formas ocorreram juntas no mesmo local. Quase todos os machos adultos observados em campo apresentaram coloração amarela, com exceção de um indivíduo alaranjado. Já as fêmeas apresentaram todos os padrões de cores citados. Cruzamentos realizados em laboratório demonstraram que indivíduos de diferentes colorações e/ou diferentes áreas copulam normalmente entre si, indicando não haver barreira mecânica ou comportamental que impeça a cópula entre as diferentes formas. Adultos de $N$. lar ocorreram o ano todo, apresentando as maiores abundâncias entre outubro e fevereiro, e as mais baixas, entre abril e agosto. Tanto a porcentagem de ocupação das plantas, quanto a densidade de indivíduos de $N$. lar nas plantas acompanharam o padrão de sua flutuação populacional. Assim, parece que $N$. lar apresenta uma estação reprodutiva por ano, entre os meses de outubro e fevereiro, época coincidente com a estação chuvosa e emissão de ramos novos pela planta de alimentação.

PALAVRAS-CHAVE. Byrsonima sericea; variação de cor; ciclo de vida; distribuição espacial; flutuação populacional.

Curculionidae é a família de Coleoptera mais rica em espécies, apresentando uma grande diversidade de hábitos, incluindo muitas espécies pragas de plantações. Por esse motivo, a maioria dos estudos visa o conhecimento de sua flutuação populacional (Ribeiro 2000; Thomazini 2002; Gopar \& Ves Losada 2004, entre outros) para o planejamento de estratégias de manejo e combate mais eficazes (Ronchi-Teles \& Silva 2005).

O gênero Naupactus possui mais de 150 espécies descritas, distribuídas na região neotropical do México à Argentina, sendo o Brasil o país que possui a maior diversidade (Lanteri et al. 2002). Besouros deste gênero são considerados pragas importantes, pois as larvas de muitas espécies são brocadoras de raiz, causando sérios danos ao sistema radicular de várias culturas, como de Citrus em São Paulo (Lanteri et al. 2002; Guedes \& Parra 2004), alfafa (Medicago sativa L.) (Ribeiro 2000; Gopar \& Ves Losada 2004) e lótus (Lotus corniculatus L.) (Ribeiro 2000) na Argentina. O ataque das larvas se dá em massa e elas podem estar localizadas a vários metros de profundidade, dificultando qualquer tipo de controle (Fernandes \& Bueno 2000).

Apesar de o gênero ser de relevante interesse econômico, pouco se conhece sobre a espécie Naupactus lar Germar, 1824. Sabe-se, apenas, que os adultos são fitófagos e polífagos, pois já foram registrados alimentando-se em Bauhinia brevipes Vogel (Leguminosae) na Estação Ecológica de Pirapitinga, MG (Cornelissen \& Fernandes 2001a, b, c), em Byrsonima sericea DC (Malpighiaceae; Flinte et al. 2006) e Manilkara subsericea 
(Mart.) Dubard (Sapotaceae; Nascimento, comunicação pessoal) no Parque Nacional da Restinga de Jurubatiba, RJ. Observações preliminares feitas neste Parque Nacional permitiram identificar muitos indivíduos de $N$. lar de diferentes cores, porém, não há na literatura nenhuma descrição de coloração ou qualquer estudo sobre aspectos ecológicos e comportamentais da espécie.

Assim, este trabalho teve como objetivo estudar, pela primeira vez, aspectos da ecologia e comportamento de $N$. lar, incluindo a descrição da variação espacial na coloração dos indivíduos da população dessa espécie no Parque Nacional da Restinga de Jurubatiba, Rio de Janeiro.

\section{MATERIALE MÉTODOS}

Área de estudo. $\mathrm{O}$ trabalho foi realizado de fevereiro de 2003 a maio de 2004 no Parque Nacional da Restinga de Jurubatiba (PNRJ), que abrange os municípios de Macaé, Carapebus e Quissamã, no Estado do Rio de Janeiro (22 ${ }^{\circ} 19^{\prime} \mathrm{S}$ e $\left.41^{\circ} 44^{\prime} \mathrm{W}\right)$. Este parque possui uma área total de 14.451 ha, e uma grande diversidade de habitats, como restinga de moitas, restinga paludosa, mata, brejos, rios, entre outros (Araújo et al. 1998).

O clima da região é tropical, apresentando verão quente e chuvoso e inverno seco e com temperaturas mais amenas (Flinte et al. 2006). Dados obtidos pela Estação Evaporimétrica Agropecuária Carapebus Ltda. demonstraram que, no período de estudo, a estação seca estendeu-se de abril a setembro de 2003, com precipitação média mensal de $65,76 \mathrm{~mm}$, enquanto que a estação chuvosa foi de outubro de 2003 a março de 2004, com precipitação média mensal de 148,03 mm. Não foi observada variação acentuada na temperatura média entre as estações seca e chuvosa $\left(26,5^{\circ} \mathrm{C}\right.$ e $25,7^{\circ} \mathrm{C}$, respectivamente).

As observações de campo foram realizadas em quatro áreas do Parque, denominadas como: 1) Lagoa de Cabiúnas (Macaé); 2) Lagoa Comprida (Carapebus); 3) Praia de Carapebus (Carapebus) e 4) Praia João Francisco (Quissamã) (Fig. 1).

Variação na coloração e biologia de Naupactus lar. Para descrever a variação espacial na coloração de N. lar no PNRJ, foram feitas duas amostragens, uma em fevereiro de $2004 \mathrm{e}$ outra em março de 2004, nas quatro áreas escolhidas. Em cada área foram vistoriados, visualmente, 30 indivíduos de Byrsonima sericea, registrando-se o número de besouros de cada sexo e sua cor. Após a inspeção visual, em cada planta, utilizou-se o método do guarda-chuva (semelhante ao descrito por Almeida et al. 1998) para complementar a amostragem dos besouros. Esta espécie de planta foi escolhida, pois é bastante abundante e amplamente distribuída no Parque, e foi considerada por Flinte et al. (2006) uma importante planta hospedeira para adultos de N. lar.

A diferenciação entre macho e fêmea foi feita visualmente no campo, considerando que as fêmeas eram maiores e tinham abdômen arredondado, enquanto que os machos eram menores que as fêmeas, e tinham o abdômen mais afinado.

No intuito de testar preliminarmente se as diferentes cores representavam diferentes formas da mesma espécie, indivíduos de ambos os sexos de cada localidade foram coletados. Em laboratório, os insetos foram colocados em potes plásticos com as seguintes combinações de área e sexo: (a) machos da área 1 com fêmeas das áreas 2 e 4, (b) machos da área 2 com fêmeas das áreas 3 e 4, e (c) machos da área 4 com fêmeas das áreas 1,2 e 3. Não foram encontrados machos na área 3. Os adultos eram alimentados com folhas frescas de $B$. sericea $\mathrm{e}$ as cópulas eram acompanhadas por observação visual três vezes ao dia, até a morte dos besouros.

Para obtenção de dados sobre o ciclo de vida da espécie, os ovos obtidos em laboratório foram colocados em potes cilíndricos de $3 \mathrm{~cm}$ de diâmetro por $3 \mathrm{~cm}$ de altura, contendo areia da restinga. Após a eclosão, foi observado que as larvas apresentavam aspecto vermiforme com mandíbulas negras, como descrito por Ribeiro (2000) para larvas da tribo Naupactini. Considerando que as larvas são brocadoras de raiz, foram alimentadas com ramos frescos de B. sericea de aproximadamente $2,7 \mathrm{~mm}( \pm 0,07)$ de diâmetro, enterrados na areia e trocados a cada dois dias, sendo anotados o tempo de duração de cada estágio (ovo, larva e pupa).

Variação temporal de Naupactus lar e padrão de distribuição em Byrsonima sericea. Para estudar a variação temporal da espécie, foram feitas vistorias bimestrais, de abril de 2003 a maio de 2004, em 120 plantas de B. sericea, sendo 30 na área 1 e 90 na área 2, nas quais anotavam-se a abundância de indivíduos de $N$. lar de cada sexo e cor e a fenologia das plantas.

A partir destes dados também foi descrito o padrão de ocupação e distribuição dos besouros na planta hospedeira,

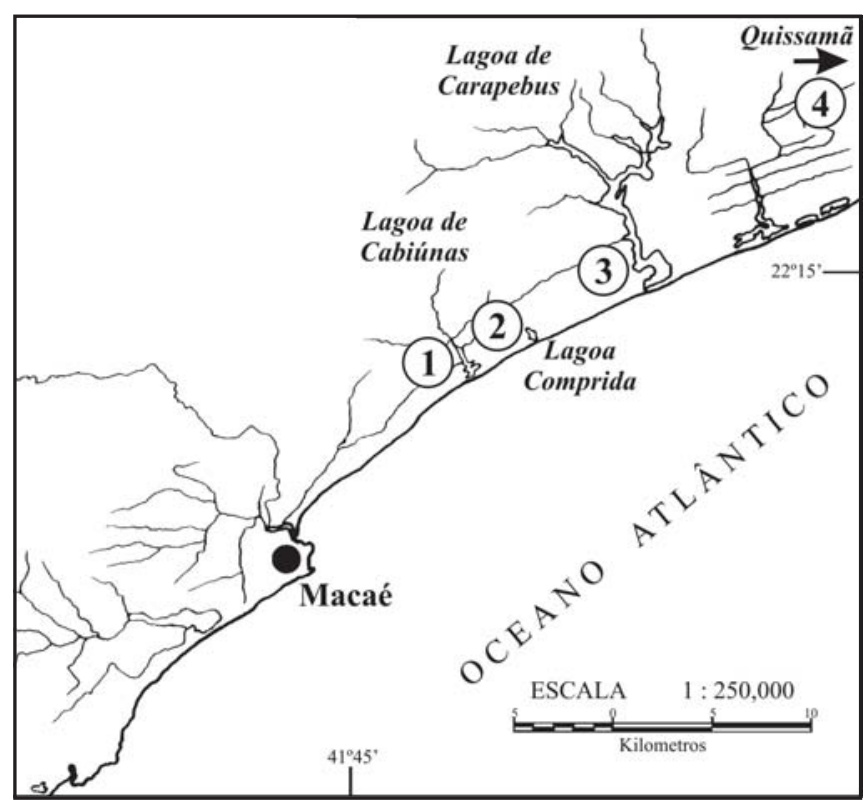

Fig. 1. Localidades onde foram realizadas as vistorias no Parque Nacional da Restinga de Jurubatiba: 1- Lagoa de Cabiúnas (Macaé), 2- Lagoa Comprida (Carapebus), 3- praia de Carapebus (Carapebus) e 4- praia de João Francisco (Quissamã). 


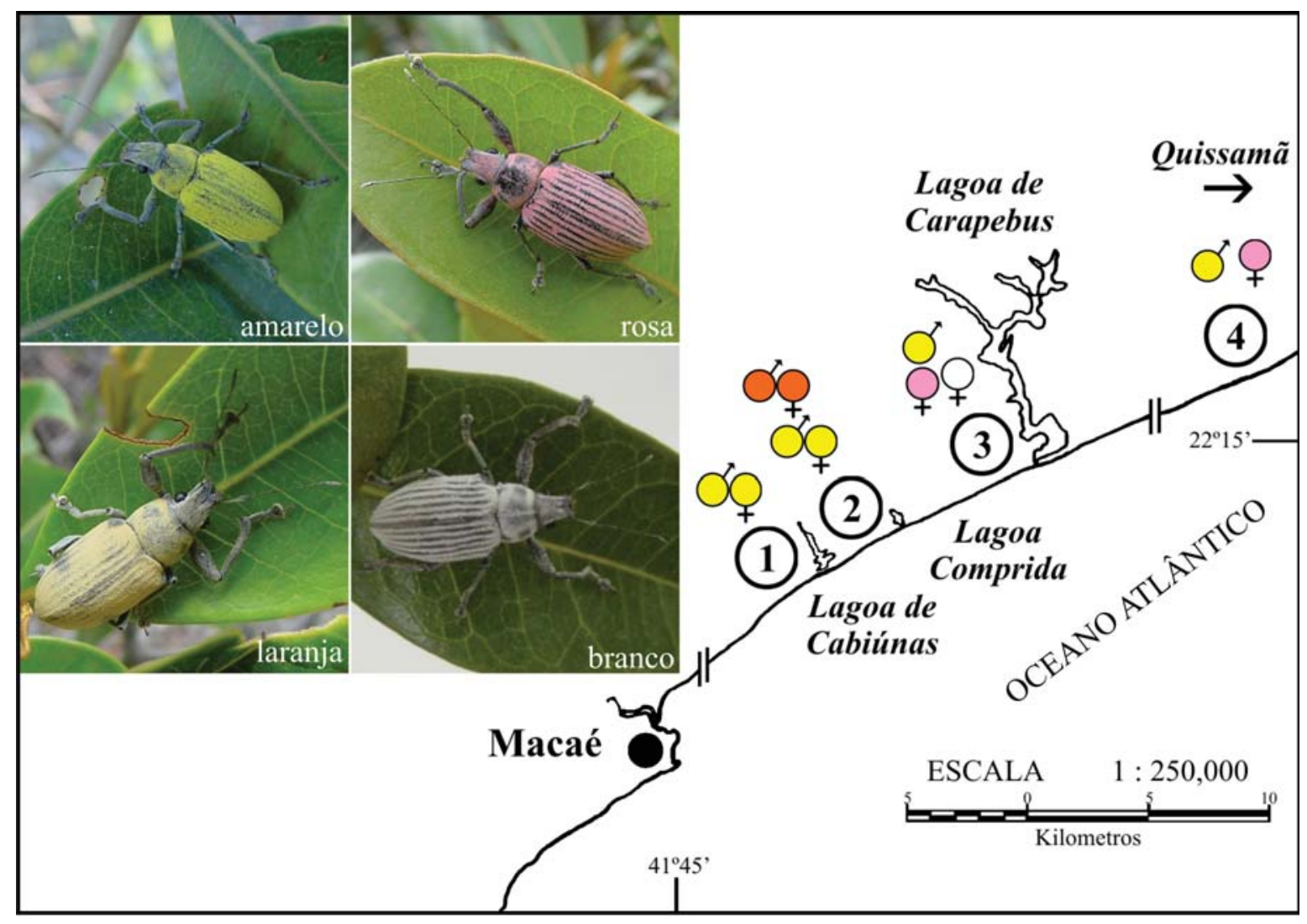

Fig. 2. Padrões de coloração de Naupactus lar e distribuição espacial das cores e dos sexos nas quatro áreas estudadas do PNRJ.

calculando-se a porcentagem de plantas ocupadas e a densidade de besouros nessas plantas. Esses dados tinham por finalidade averiguar se havia alguma relação entre estas variáveis ao longo do período de estudo, bem como em relação às características fenológicas das plantas.

\section{RESULTADOSEDISCUSSÃO}

Variação espacial e sexual da coloração de Naupactus lar. Naupactus lar apresentou quatro formas diferentes em termos de coloração no PNRJ. Todos os machos observados tinham a coloração amarela, exceto um indivíduo de coloração alaranjada encontrado na área 2 , enquanto as fêmeas exibiram as cores laranja, branco e rosa, além do amarelo (Fig. 2).

O padrão de coloração das fêmeas de $N$. lar distribuiu-se de forma diferente nas quatro áreas vistoriadas: na área 1 foram encontradas fêmeas amarelas $(\mathrm{N}=8)$, havendo apenas uma fêmea laranja. $\mathrm{Na}$ área 2 haviam tanto fêmeas amarelas $(\mathrm{N}=9)$ quanto laranjas $(\mathrm{N}=11)$. Já na área 3 foram encontradas fêmeas brancas $(\mathrm{N}=4)$ e rosas $(\mathrm{N}=7)$, e na área 4 foram encontradas somente fêmeas rosas ( $\mathrm{N}=27$ ) (Fig. 3). Isso sugere a existência de um gradiente de substituição de cores na população do sul para o norte.

Os cruzamentos realizados em laboratório com as diferentes combinações de área e sexo mostraram que todos os indivíduos testados, mesmo de cores e locais diferentes, copularam normalmente entre si, indicando não haver barreira mecânica ou comportamental impedindo a cópula entre as diferentes formas. Este resultado indica que as diferentes cores representam formas diferentes da mesma espécie.

O policromatismo em insetos em restingas do Estado do Rio de Janeiro já foi registrado para algumas espécies. Monteiro (1990) encontrou que larvas de duas espécies de borboletas do gênero Rekoa (Lycaenidae) apresentavam cores crípticas bem distintas, dependendo da cor do broto floral do qual se alimentavam, sendo um policromatismo adaptativo contra predadores visualmente orientados. Gonçalves \& Macedo (2003) encontraram que Chelymorpha cribraria Fabricius, 1775 (Cassidinae) apresenta diferentes formas, representadas por combinações de coloração do pronoto e do élitro. Grenha et al. (2004) observaram que o besouro Mecistomela marginata Thunberg, 1821 apresentou variação geográfica na coloração das populações com uma cor (vermelha) predominante no sul e outra (amarela) mais comum no norte e no interior da mata atlântica.

No caso de $N$. lar, embora não se saiba se a origem da variação da cor é fenotípica ou genotípica, essa divisão da população em diferentes formas, levando a uma diversidade de aspectos, poderia ser uma característica adaptativa, dificultando a imagem de busca de possíveis predadores visualmente orientados, como sugerido por Monteiro (1990) para o policromatismo das larvas do gênero Rekoa. 


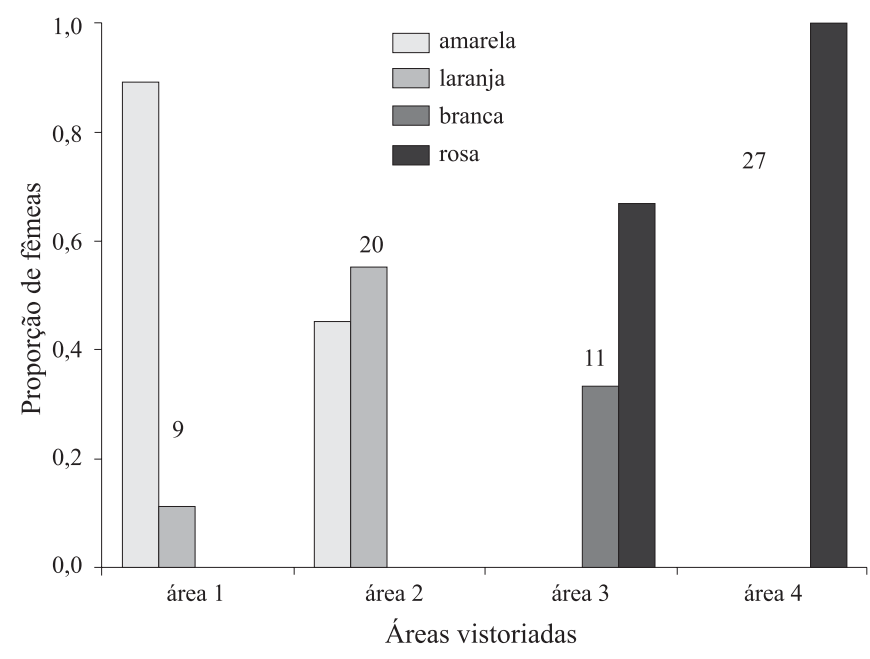

Fig. 3. Proporção de fêmeas de Naupactus lar de cada cor nas quatro áreas estudadas no PNRJ (barras) e total de fêmeas por área (número sobre as barras).

Biologia de Naupactus lar. Todas as fêmeas mantidas em laboratório $(n=10)$ colocaram, juntas, 250 ovos durante o período de criação. As larvas resultantes dessa criação mostraram-se receptivas à alimentação, raspando os ramos de B. sericea, com o qual obtinham grande crescimento corporal. Apesar do grande número inicial de larvas $(\mathrm{n}=250)$, somente um indivíduo completou seu desenvolvimento, emergindo um macho amarelo. A alta taxa de mortalidade na fase larval pode ter ocorrido por fatores como a falta de condições de temperatura e umidade adequadas e/ou pela freqüiente infecção por fungos verificada na criação. Ribeiro (2000) obteve uma mortalidade larval de até $90 \%$ em várias espécies da tribo Naupactini, devido, principalmente, a infecções por fungos dos gêneros Beauveria e Metarhizium, que são organismos amplamente utilizados no controle biológico de outros insetos. A mesma autora cita o canibalismo como uma característica da tribo desse besouro quando as larvas estão em altas densidades, o que também pode ter contribuído para a alta taxa de mortalidade, resultando em indivíduos isolados ao final da criação, fato observado por essa autora e no presente estudo.

O único adulto obtido em laboratório teve um tempo total de desenvolvimento de oito meses e seis dias, sendo 16 dias para a eclosão do ovo, sete meses de desenvolvimento larval e 20 dias como pupa, tempo semelhante ao descrito por diversos autores para espécies da tribo Naupactini (e.g. Ribeiro 2000; Peña 2001; Lanteri et al. 2002). As outras larvas criadas $(n=249)$ levaram $16( \pm 3)$ dias para eclodir do ovo, mas não chegaram à fase adulta, morrendo, na maioria das vezes, antes do quinto mês de desenvolvimento.

Flutuação populacional. Adultos de $N$. lar ocorrem durante todo o ano, apresentando abundâncias mais altas entre outubro e fevereiro, e mais baixas entre abril e agosto na espécie de planta vistoriada (Fig. 4). Tal padrão temporal, juntamente com o ciclo de vida longo, sugere que $N$. lar apresenta uma só estação reprodutiva por ano com pico populacional no mês de outubro, início da primavera.

Três estudos com outras espécies de Naupactus encontraram padrões populacionais semelhantes aos do presente estudo. Ribeiro (2000) detectou variação na abundância populacional de três espécies de Naupactus no Uruguai, ao longo dos dois anos estudados, com um pico populacional por ano, concentrados no verão. Lanteri et al. (2002) demonstraram, para outras espécies do gênero Naupactus em São Paulo, a ocorrência de indivíduos ao longo de todo o ano, declinando somente no inverno. Por fim, Gopar \& Ves Losada (2004) observaram o início da emergência de adultos de Naupactus leucoloma Boheman na Argentina em novembro, com pico de adultos em dezembro. Assim, esses estudos sugerem que a estação reprodutiva das espécies de Naupactus tende a ocorrer uma vez ao ano, nos meses mais quentes e úmidos, quando é encontrado o maior número de indivíduos.

No caso de $N$. lar, uma possível explicação para sua dinâmica é que a espécie pode estar sendo afetada direta e
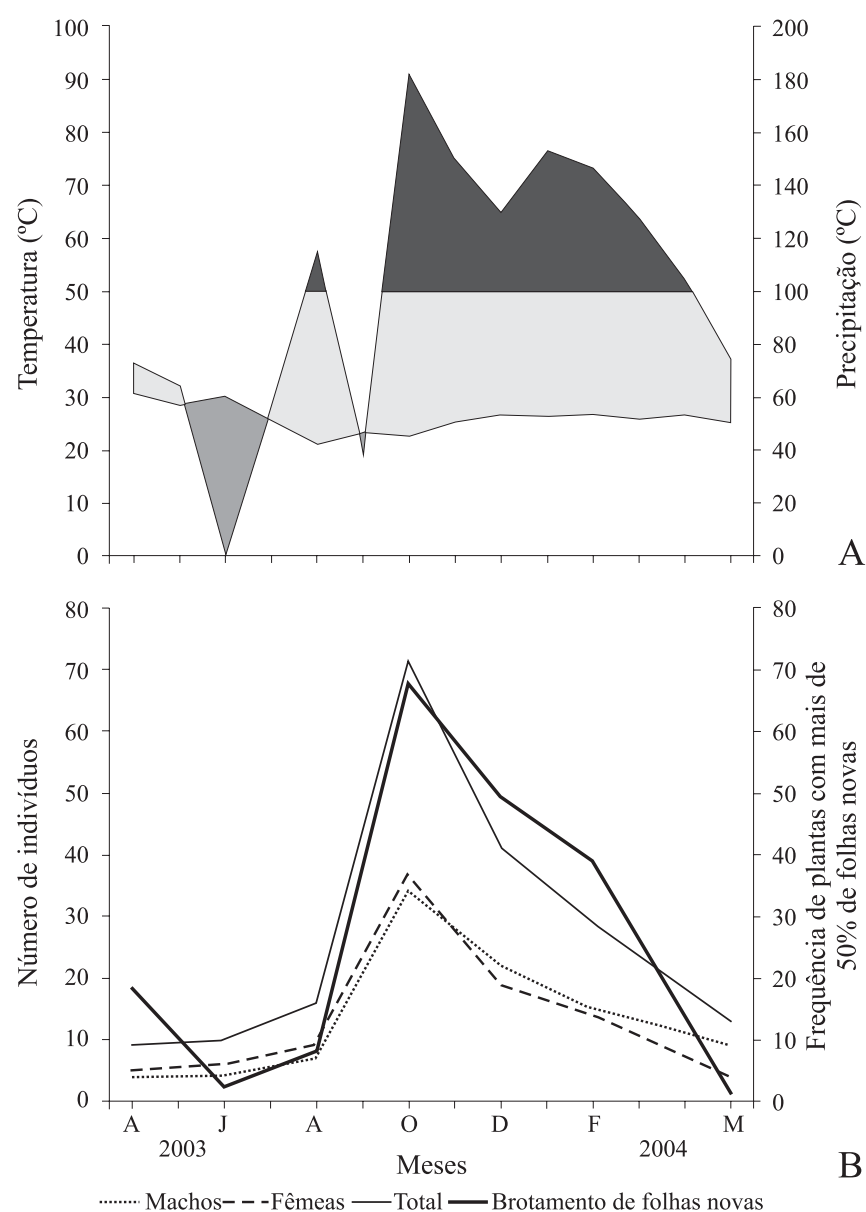

Fig. 4. Diagrama climático da área de estudo (A) e variação na abundância de machos e fêmeas de Naupactus lar em relação à freqüência (\%) de plantas com mais de $50 \%$ de suas folhas em estado jovem (B), de abril de 2003 a maio de 2004. Área pontilhada= período seco; área hachurada= período úmido; área cheia= período superúmido. 


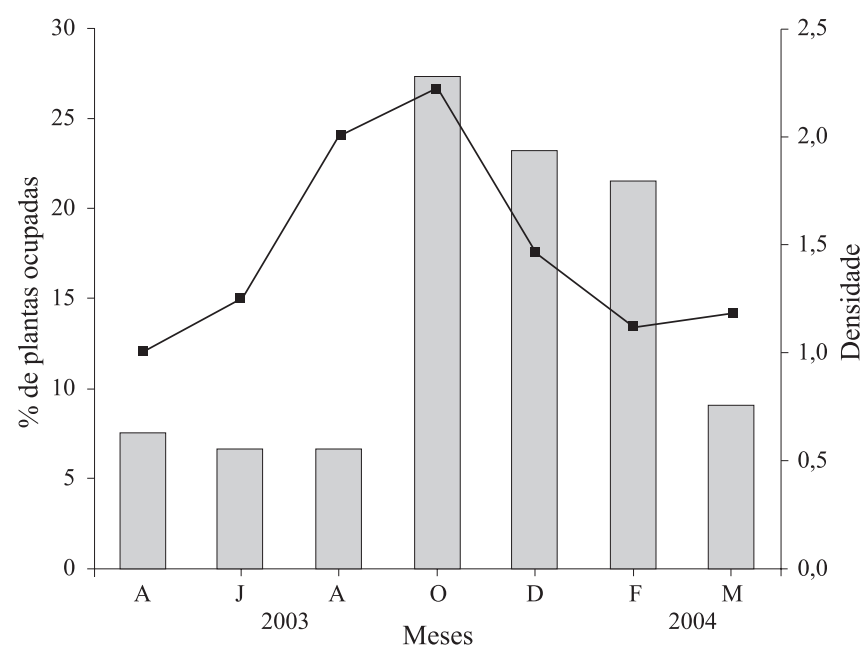

Fig. 5. Variação temporal na porcentagem de plantas ocupadas (barras) e na densidade de indivíduos de Naupactus lar nestas plantas (linha) nas áreas 1 e 2, no período de abril de 2003 a maio de 2004.

indiretamente pela estação chuvosa, já que seu crescimento populacional ao longo do tempo coincide com o aumento de precipitação e crescimento vegetativo da planta hospedeira, B. sericea (Fig. 4). Como foram vistos poucos adultos na estação seca, é possível que a maior parte da população esteja na fase larval, brocando as raízes, e os adultos existentes estejam ocupando outras espécies de plantas hospedeiras e/ ou sejam raros nessa época. $\mathrm{O}$ aumento de indivíduos adultos na primavera sugere que estejam emergindo e/ou mais ativos nesta época, devido à chegada da estação chuvosa e emissão de ramos novos pelas plantas de alimentação.

Padrão de distribuição nas plantas. De fevereiro a agosto, menos de $10 \%$ dos 120 indivíduos de B. sericea vistoriados apresentaram adultos, enquanto que, a partir do mês de setembro, a porcentagem de plantas ocupadas por adultos aumentou, chegando a uma média de $28 \%$ nos meses de outubro e dezembro (Fig. 5).

Além do aumento do número de plantas com besouros, houve também um aumento na densidade de adultos nessas plantas. A densidade média de $N$. lar nas plantas ocupadas foi de 1 indivíduo por planta na estação seca e 2,2 indivíduos por planta na estação chuvosa (Fig. 5). Dessa forma, supõe-se que estes besouros estão ocupando mais plantas e, ao mesmo tempo, se agregando mais durante a época de maior abundância de adultos.

Alguns trabalhos de distribuição espacial de espécies pragas de Curculionidae mostram que nas culturas que atacam, mantêm uma distribuição agregada em todo o ano, apesar de haver períodos de maior e menor atividade (Faleiro et al. 2002; Oehlschlager et al. 1995). Tal padrão de agregação ao longo de todo o ano não foi observado em $N$. lar, possivelmente porque sua população não tenha atingido densidades tão altas na restinga, mesmo em $B$. sericea, além de ocorrerem, embora mais raramente, em outras espécies de plantas hospedeiras. Uma hipótese para a agregação é que, com a chegada da primavera e emissão de ramos com folhas novas por B. sericea, muitas dessas plantas podem oferecer características nutritivas mais adequadas para a alimentação dos adultos de $N$. lar e, com isso, os adultos passam a ocupar mais plantas desta espécie e se agregam mais neste período.

Outra hipótese para a agregação, que não exclui a hipótese anterior, seria o aumento da chance de encontro de parceiros na fase reprodutiva. Faleiro et al. $(1998,1999)$ sugerem a existência de feromônios de atração sexual no gorgulho Rhynchophorus ferrugineus Olivier, 1790, levando à agregação na fase reprodutiva, porém, tal aspecto não foi estudado para N. lar.

Apesar destas hipóteses, é necessário um conhecimento mais detalhado sobre a biologia e ecologia do inseto para explicar a agregação e entender quais fatores estariam influenciando neste comportamento. Tais estudos poderiam também ajudar a elucidar se a variação encontrada no número de besouros é um aumento populacional real, ou se representa somente um aumento da atividade e/ou da agregação desses insetos sobre a planta hospedeira estudada.

A variação de coloração em $N$. lar, observada tanto espacialmente quanto em relação ao sexo, foi registrada pela primeira vez nesse trabalho. Entretanto, estudos mais detalhados e experimentais sobre ecologia e genética, abrangendo uma área mais extensa de sua distribuição, poderiam contribuir para a descrição e significado adaptativo do policromatismo e das variações espaço-temporais.

Agradecimentos. Ao Dr. Sérgio Vanin (Depto. de Zoologia/USP) pela identificação do besouro, ao CNPq e CNPq/PELD (Pesquisas Ecológicas de Longa Duração) pelo financiamento do trabalho e bolsa de produtividade (RFM), à CAPES pela bolsa de doutorado (VF) e mestrado (COA), ao IBAMA/PNRJ pela licença para pesquisa e à equipe do Laboratório de Ecologia de Insetos (UFRJ) pela ajuda no campo. Agradecemos também aos revisores, que fizeram contribuições importantes para melhoria desse manuscrito.

\section{REFERÊNCIAS}

Almeida, L. M.; C. S. Ribeiro-Costa \& L. Marinoni. 1998. Manual de coleta, conservação, montagem e identificação de insetos. Ribeirão Preto, Holos, 78 p.

Araújo, D. S. D.; F. R. Scarano; C. F. C. Sá; B. C. Kurtz; H. L. T. Zaluar; R. C. M. Montezuma \& R. C. Oliveira. 1998. Comunidades vegetais do Parque Nacional da Restinga de Jurubatiba, p. 39-62. In: F. A. Esteves (ed.). Ecologia das Lagoas costeiras do Parque Nacional da Restinga de Jurubatiba e do Município de Macaé (RJ). Rio de Janeiro, NUPEM-UFRJ, 464 p.

Cornelissen, T. G. \& G. W. Fernandes. 2001a. Defence, growth and nutrient allocation in the tropical shrub Bauhinia brevipes (Leguminosae). Austral Ecology 26: 246-253.

Cornelissen, T. G. \& G. W. Fernandes. 2001b. Induced defenses in the neotropical tree Bauhinia brevipes (Vog.) to herbivory: effects of damage-induced changes on leaf quality and insect attack. Tree 15: 236-241.

Cornelissen, T. G. \& G. W. Fernandes. 2001c. Patterns of attack by herbivores on the tropical shrub Bauhinia brevipes (Leguminosae): Vigour or chance? European Journal of Entomology 98: 3740 .

Faleiro, J. R.; V. A. Abrahan \& M. A. Al Shuaibi. 1998. Role of pheromone trapping in the management of red palm weevil 
Indian Coconut Journal 29: 1-3.

Faleiro, J. R.; M. A. Al Shuaibi; V. A. Abraham \& T. Ferem Kumar. 1999. A technique to asses the longevity of the pheromone (Ferrolure) used in trapping the date red palm weevil Rhynchophorus ferrugineus Oliv. Agricultural Science 4: 5-9.

Faleiro, J. R.; J. Ashok Kumar \& P. A. Rangnekar. 2002. Spatial distribution of red palm weevil Rhynchophorus ferrugineus Oliv. (Coleoptera: Curculionidae) in coconut plantations. Crop Protection 21: 171-176.

Fernandes, O. A. \& A. F. Bueno. 2000. Manejo Integrado de Pragas: Tópicos práticos para algumas culturas. Jaboticabal, FCAV/ UNESP, 94 p. (www.fcav.unesp.br/apecolab), acesso em: 12/08/ 2006.

Flinte, V.; C. O. Araujo; M. V. De Macedo \& R. F. Monteiro. 2006. Insetos fitófagos associados ao murici da praia, Byrsonima sericea (Malpighiaceae), na Restinga de Jurubatiba (RJ). Revista Brasileira de Entomologia 50: 512-523.

Gonçalves, R. O. \& M. V. Macedo. 2003. Population ecology of the polymorphic species Chelymorpha cribraria (Col.: Chrysomelidae) in Rio de Janeiro, Brazil, p. 285-294. In: D. G. Furth (ed.). Special Topics in Leaf Beetle Biology. Proceedings of the Fifth International Symposium on the Chrysomelidae. SofiaMoscow, Pensoft Publishers, xii +339 p.

Gopar A. \& J. C. Ves Losada. 2004. Publicaciones Técnicas $n^{\circ}$ 57: Estudios sobre la fluctuación poblacional de gorgojos (Coleoptera: Curculionidae) adultos que afectan a la alfalfa (Medicago sativa, L.). La Pampa, INTA, $20 \mathrm{p}$.

Grenha, V.; M. V. Macedo \& R. F. Monteiro. 2004. Geographical variation in Mecistomela marginata (Hispinae), p. 225-230. In: P. H. Jolivet, J. A. Santiago-Blay \& M. Schmitt. (eds.). New developments in the Biology of Chrysomelidae. The Hague,
SPB Academic Publishers, $x x+803$ p.

Guedes, J. V. C. \& J. R. P. Parra. 2004. Ovoposição dos curcúlios-dasraízes dos citrus (Coleoptera: Curculionidae). Ciência Rural 34: 673-678.

Lanteri, A. A.; J. C. Guedes \& J. R. P. Parra. 2002. Weevils injurious for citrus in São Paulo state, Brazil. Neotropical Entomology 31: $561-569$.

Monteiro, R. F. 1990. Cryptic larval polychromatism in Rekoa marius Lucas and $R$. palegon Cramer (Lycaenidae: Theclinae). Journal of Research on the Lepidoptera 29: 77-84.

Oehlschlager, A. C.; R. S. McDonald; C. M. Chinchilla \& S. N. Patschke. 1995. Influence of pheromone based mass trapping system on the distribution of Rhynchophorus palmarum (Coleoptera: Curculionidae) in oil palm. Environmental Entomology 24: 10051012.

Peña, L. A. V. 2001. Gusanos blancos de la papa: biología e manejo. Revista Corpoica: Innovación y Cambio Tecnológico 2: 2933.

Ribeiro, A. 2000. Estudios Preliminares Sobre Poblaciones de Gorgojos de Suelo en Pasturas de Alfalfa y Lotus. In: O. Ernst; F. GarcíaPréchac \& D. Martino (eds.) Curso de Actualización para Profesionales Universitarios: Siembra Sin Laboreo de Cultivos y Pasturas. Montevideo, INIA, PROCISUR, EEMAC. (http://www.fagro.edu.uy/ eemac/Siembra\%20Directa/6B.pdf), acesso em: 24/08/2006.

Ronchi-Teles, B. \& N. M. Silva. 2005. Flutuação populacional de espécies de Anastrepha Schiner (Diptera: Tephritidae) na região de Manaus, AM. Neotropical Entomology 34: 733-741.

Thomazini, M. J. 2002. Flutuação populacional e intensidade de infestação de broca-dos-frutos em cupuaçu. Scientia Agricola 59: $463-468$. 